\title{
Dynamic process migration framework
}

\begin{abstract}
Process migration refers to the act of transferring a process in the middle of its execution in a network. The majority of today's computing power exists in the form of workstations. Increasing use of autonomous workstations connected by a high-speed network represents substantial opportunity for sharing more resources and designing a highly available system much cheaper than a high performance single machine. The process migration framework as a bare facility should be included in all Operating Systems (OSs). In this paper, we describe the design and implementation of process migration framework for Linux OS which aims to segregate the process migration mechanism from the system design and provide the capability of dynamically extending the process migration system on demand to increase compatibility and reduce the system overhead.
\end{abstract}

Keyword: Process migration mechanism; Migration framework 